\title{
Evaluation of Assistive Devices Using Humanoid Robot with Mechanical Parameters Identification
}

\author{
Ko Ayusawa ${ }^{1}$, Shin'ichiro Nakaoka ${ }^{2}$, Eiichi Yoshida ${ }^{1}$, Yumeko Imamura ${ }^{3}$ and Takayuki Tanaka ${ }^{3}$
}

\begin{abstract}
This paper presents the application of a humanoid robot as an evaluator of assistive devices; we propose a framework of the evaluation by utilizing identification of the mechanical properties of a humanoid robot. The accurate estimation of joint torque with the identification can enhance the performance to estimate the supporting effect of the devices. We evaluate a passive assistive wear "Smart Suit Lite (SSL)" as an example of device, and use HRP-4C as the humanoid platform. With the general formulation of the wire-driven multi-body system, the supporting torque of passive assistive devices is also formulated. The identification of the stiffness of SSL is performed an example of the evaluation with HRP-4C, and the result is compared to the ground truth value.
\end{abstract}

\section{INTRODUCTION}

Human assistive devices are expected to support daily life under the super-aged societies in several nations, and are studied and developed intensively [1], [2]. The quantitative criteria or standard for the safety evaluation and the assistive performance will have a great role, in order to promote the industrial growth and expansion in this field. The measurement of human subjects is one way to evaluate the devices. The recent techniques of human motion analysis enables the estimation of the somatosensory information [3]; on the other hand, the quantitative evaluation of the devices by human measurement still has the problems like: variation of human subjects, gathering appropriate subjects, reproducing the same motions, ethical procedures for the experiments with unevaluated devices, and so on. The estimation methodology of the external contact forces or muscle tensions is always confronted with the redundancy problem [3], because of the difficulty of their direct measurement. Especially when the subject wears the suit-type device, it is of great difficulty to measure or extract the force effects of the device on the body. Humanoid robots with their internal sensors are, instead, expected to provide the conventional evaluation scheme with the additional information about internal forces [4], since they are uninfluenced by the above issues.

When evaluating the devices with a humanoid robot, the following issues have to be considered.

A) The humanoid robot is required to have almost the same morphology as a human, because the robot has to use

*This research was partly supported by JSPS KAKENHI Grant Number 25820082, and by METI Robotic Devices for Nursing Care Project.

${ }^{1} \mathrm{~K}$. Ayusawa and E. Yoshida are with CNRS-AIST JRL (Joint Robotics Laboratory), UMI3218/CRT, and ${ }^{2}$ Shin'ichiro Nakaoka is with Humanoid Research Group, both Intelligent Systems Research Institute, National Institute of Advanced Industrial Science and Technology (IS-AIST), Japan $\{k$.ayusawa, s.nakaoka, e.yoshida\}@aist.go.jp

${ }^{3}$ Y. Imamura and T. Tanaka are with Graduate School of Information Science and Technology, Hokkaido University, Japan the devices originally designed for human morphology. The correspondence of body parts also makes it easier to validate the effect on humans from the results of humanoid robots.

B) The techniques of human motion retargeting to a humanoid robot are necessary; the robots have to perform same motion as humans do, which meets objectives of the evaluation of the devices for humans.

C) The accurate measurement or estimation especially of joint torque is required. Joint torque is an important mechanical quantity when evaluating the assistive devices, since the role of many devices is to reduce the load applied on joints.

HRP-4C [5] is one of humanoid robots that can overcome the first issue. The geometric parameters of the robot are designed close to the measured average of Japanese females. Some studies related to the second issue demonstrated their created motion on a humanoid robot with human motion data [6], [7]. They are adapted to reproduction of whole-body motion preserving many kinematic and dynamic constraints for the humanoid. It should be noted that, strictly speaking, its joint configuration is still different from that of the actual human body. However, the human model used in the inverse dynamics analysis is also often simplified [8], [9] because of some practical issues. Those simplified models are actually used in biomechanics, for instance to estimate the load in lower back [10]. The joint configuration of HRP-4C is at least nearly equal to that of those models.

Although some basic estimation of supportive effects from motor torque has been reported in [4], concerns about the accuracy of joint torque measurement (the issue $\mathrm{C}$ ) above) still remained. The recent torque sensors become so compact as to be installed on a humanoid robot [11]; however, it still requires time to realize weight saving and downsizing in order to keep human morphology for our application. Since we should rely on the input of electric motors, we propose a method for estimating joint torques to take into account uncertainties and changes (e.g. carrying an object) in mechanical properties or the robots.

In this paper, we propose the framework of the evaluation by utilizing the identification of mechanical properties of a humanoid robot. The method to identify simultaneously the parameters of the whole body dynamics, the actuator model, and the friction model is proposed. It is based on the identification of the inertial parameters of a humanoid robot [12]. As an example of assistive device, we evaluate a passive assistive wear "Smart Suit Lite (SSL)" that supports the load at the lower back with the elastic bands [2]. We show 
the general modeling to compute the supporting torques of those passive assistive wears by using the same formulation of the musculoskeletal computation [3]. We also present the two approaches to estimate the supporting torque from the sensor data of a humanoid robot. Finally, the identification of the stiffness of the device is performed. The result is validated and compared to the ground truth value obtained of the component test of the device.

\section{FLOW OF EVALUATION WITH HUMANOID}

When evaluating passive assistive devices with a humanoid robot, if the same body structure, motion and the contact situation as a human are ideally realized, it can lead exactly the same mechanical state of the device as when a human wears the device. By recording the values of the internal sensors, the mechanical properties and states of the device during the motion can be identified and estimated.

Under this assumption, it is essential that the humanoid robot has to be almost the same morphology as a human. HRP-4C [5] is one of the humanoid robots which fulfill the requirement; the robot is $1.58 \mathrm{~m}$ in height and $43 \mathrm{~kg}$ in weight, featuring its geometric parameters close to the measured average of women of 19-29 years old in Japan.

Here is the evaluation flow using HRP-4C (Fig.1):

S1. Preparation of some human motion data.

S2. Human motion retargeting to the humanoid robot.

S3. Identification of the mechanical parameters of the robot by experimental data.

S4. Estimation of supporting torques of the devices.

S5. Evaluation of the devices.

Even though we evaluate the devices with a humanoid robot, we have to define the human motion which should be performed in the evaluation. In order to prepare them in step 1(S1), for example, we utilize the human motion capturing. The detail of this procedure is shown in [4].

In step 2, the motion of the humanoid robot is generated by using the human motion data. The generated motion has to preserve the kinematic and dynamic characteristics of the original motion. In this paper, we use an efficient retargeting technique [4], [7], which is adapted to reproduction of whole-body motion preserving many kinematic and dynamic constraints for the humanoid.

The accurate measurement or estimation of joint torques is important, which usually requires the joint torque sensors or the identification of the mechanical parameters of each joint. Even though the recent development of the torque sensors enable them on a humanoid robot [11], it still take a time to decrease the weight and size of them in order to keep human morphology in our application. The joint torques can be estimated from the inertial parameters, motor constants, gearratio, etc. provided by manufacturers. However, they are not necessarily accurate because of the uncertain elements like frictions, and can change when, for example, robot carries the objects, the temperature condition of actuators changes, etc. We thus identify those parameters of the robot in step 3 . The detail of the identification procedure is shown in Section III.
The equations of motion of the robot with the device are simplifed as follows:

$$
\boldsymbol{\tau}(\boldsymbol{x}, \boldsymbol{\phi})=\boldsymbol{\tau}_{a c t}+\boldsymbol{\tau}_{\text {support }}
$$

where, $\boldsymbol{\tau}_{\text {act }}$ and $\boldsymbol{\tau}_{\text {support }}$ represents the actuator torque and the supporting torque respectively, $\boldsymbol{x}$ means the robot's state like joint angles and their derivatives, and $\phi$ represents the mechanical parameters of the robot. There are two approaches to estimate the supporting torques in step 4 .

(A). Perform the same motion with and without the device respectively (i.e. $\boldsymbol{x}_{\text {with }} \approx \boldsymbol{x}_{\text {without }}$ ), and compute the difference of joint torque between with and without the device:

$$
\boldsymbol{\tau}_{\text {support }}^{\text {with }}=\boldsymbol{\tau}_{\text {act }}^{\text {with }}-\boldsymbol{\tau}\left(\boldsymbol{x}^{\text {with }}, \boldsymbol{\phi}\right) \approx \boldsymbol{\tau}_{\text {act }}^{\text {with }}-\boldsymbol{\tau}_{\text {act }}^{\text {without }}
$$

where, $*^{\text {with }}$ and $*^{\text {without }}$ mean the variable when with and without the device respectively, and $\tau_{\text {support }}^{\text {without }}=\mathbf{0}$.

(B). Compute the difference between the measured joint torques and those estimated from the mechanical parameters which were identified in advance:

$$
\boldsymbol{\tau}_{\text {support }}=\boldsymbol{\tau}_{\text {act }}-\boldsymbol{\tau}(\boldsymbol{x}, \boldsymbol{\phi}) \approx \boldsymbol{\tau}_{\text {act }}-\boldsymbol{\tau}(\boldsymbol{x}, \widehat{\boldsymbol{\phi}})
$$

where, $\widehat{\phi}$ is the estimated mechanical parameters of the robot without the device.

The strong merit of approach (A) is cancelling the unmodeled friction forces; on the other hand, the same motion has to be performed between with and without the device. Some devices are originally designed so that the human should perform the different motion with and without them Approach (B) is the general formulation; the accurate model of the robot (i.e. the identification of the parameters) has to be required. Unlike approach (A), the supporting torque can be extracted directly from the recorded data with the device by approach (B).

In this paper, the performance of both approaches is investigated by experiments.

Finally, in step 5, the assistive device is evaluated by the obtained supporting torques. In this paper, we evaluate a passive assistive wear "SSL" [2], and identify the stiffness of SSL band from the experimental data of the robot. The detail of the modeling of SSL and the identification procedure is shown in Section IV.

\section{IDENTIFICATION OF HUMANOID ROBOT}

\section{A. Basic formulation}

The equations of motion of a humanoid robot are given by [13]:

$$
\left[\begin{array}{ll}
\boldsymbol{H}_{o o} & \boldsymbol{H}_{o j} \\
\boldsymbol{H}_{j o} & \boldsymbol{H}_{j j}
\end{array}\right]\left[\begin{array}{l}
\ddot{\boldsymbol{q}}_{o} \\
\ddot{\boldsymbol{q}}_{j}
\end{array}\right]+\left[\begin{array}{c}
\boldsymbol{b}_{o} \\
\boldsymbol{b}_{j}+\boldsymbol{\tau}_{f}
\end{array}\right]=\left[\begin{array}{l}
\mathbf{0} \\
\boldsymbol{\tau}
\end{array}\right]+\sum_{k=1}^{N_{c}}\left[\begin{array}{c}
\boldsymbol{K}_{o k}^{T} \\
\boldsymbol{K}_{j k}^{T}
\end{array}\right] \boldsymbol{F}_{k}^{e x t}
$$

where,

- $N_{J}$ is the number of degree of freedom (DOF),

- $\boldsymbol{q}_{o} \in S E(3)$ is the vector of the generalized coordinates which represent the position and orientation of the baselink, 


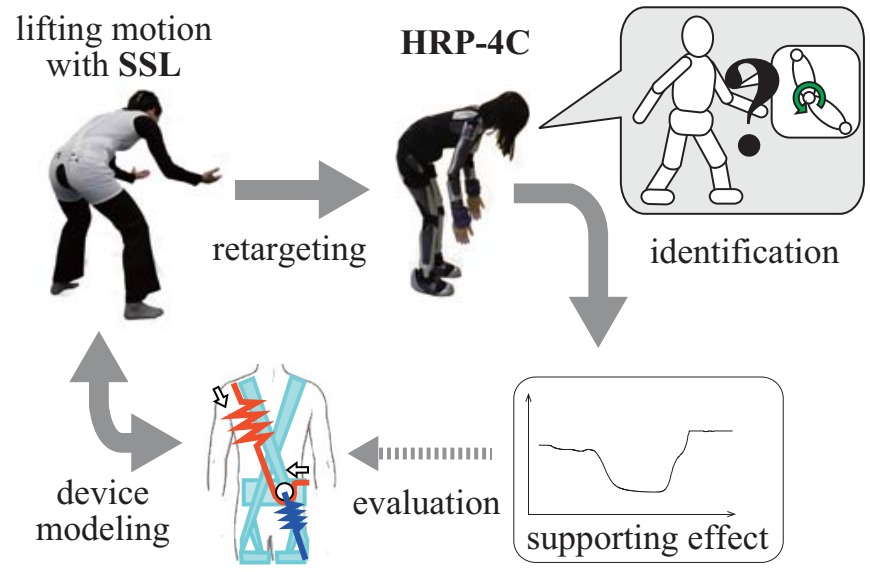

Fig. 1. Outline of the processes to evaluate assistive devices by using a humanoid robot

- $\boldsymbol{q}_{j} \in \mathbb{R}^{N_{J}}$ is the joint angle vector,

- $\boldsymbol{H}_{o o}, \boldsymbol{H}_{o c}, \boldsymbol{H}_{c o}, \boldsymbol{H}_{c c}$ are the inertia matrices,

- $\boldsymbol{b}_{o}, \boldsymbol{b}_{j}$ are the bias force vectors including centrifugal, Coriolis, and gravity, and forces,

- $\boldsymbol{\tau} \in \mathbb{R}^{N_{J}}$ is the vector of joint torque,

- $\boldsymbol{\tau}_{f} \in \mathbb{R}^{N_{J}}$ is the vector of joint friction torques,

- $N_{c}$ is the number of contact points with the environment,

- $\boldsymbol{F}_{k}^{\text {ext }} \in \mathbb{R}^{6}$ is the vector of external forces exerted to the system at contact $k$,

- $\boldsymbol{K}_{k} \triangleq\left[\begin{array}{ll}\boldsymbol{K}_{o k} & \boldsymbol{K}_{c k}\end{array}\right] \in \mathbb{R}^{6 \times\left(6+N_{J}\right)}$ is the Jacobian matrix associated to contact $k$ and of the orientation of the contact link with respect to generalized coordinates.

Let $\boldsymbol{\tau}_{f}$ contain only Coulomb and viscous frictions:

$$
\boldsymbol{\tau}_{f}=\operatorname{sgn}\left(\operatorname{diag}\left(\dot{\boldsymbol{q}}_{j}\right)\right) \boldsymbol{\tau}_{c}+\operatorname{diag}\left(\dot{\boldsymbol{q}}_{j}\right) \boldsymbol{k}_{d}
$$

where, $\boldsymbol{\tau}_{c} \in \mathbb{R}^{N_{J}}$ is the vector of Coulomb frictions, and $\boldsymbol{k}_{d} \in$ $\mathbb{R}^{N_{J}}$ is the vector of viscous friction coefficients.

Let us assume that $\tau$ is defined as the following linear identification model of motors:

$$
\operatorname{diag}(\boldsymbol{u}) \boldsymbol{k}_{T}=\boldsymbol{\tau}
$$

where, $\boldsymbol{u} \in \mathbb{R}^{N_{J}}$ is the vector of electrical currents, and $\boldsymbol{k}_{T} \in$ $\mathbb{R}^{N_{J}}$ is the vector of motor constants.

The equations of motion of multi-body systems can be written in a linear form with respect to the inertial parameters [14], [15], and Eq.(7) can be transformed from Eq.(4).

$$
\left[\begin{array}{ll}
\boldsymbol{Y}_{B o} & \boldsymbol{O} \\
\boldsymbol{Y}_{B c} & \boldsymbol{Z}
\end{array}\right]\left[\begin{array}{c}
\boldsymbol{\phi}_{B} \\
\boldsymbol{\xi}
\end{array}\right]=\left[\begin{array}{l}
\boldsymbol{F}_{o} \\
\boldsymbol{F}_{j}
\end{array}\right] \triangleq \sum_{k=1}^{N_{c}}\left[\begin{array}{c}
\boldsymbol{K}_{o k}^{T} \\
\boldsymbol{K}_{o k}^{T}
\end{array}\right] \boldsymbol{F}_{k}^{e x t}
$$

where,

- $\phi_{B} \in \mathbb{R}^{N_{B}}$ is the vector of the minimum set of inertial parameters (or base parameters) which represents the equations of motions [16], [17],

- $\boldsymbol{\xi} \triangleq\left[\boldsymbol{k}_{T}{ }^{T} \boldsymbol{k}_{d}{ }^{T} \boldsymbol{\tau}_{c}{ }^{T}\right]^{T}$ is the vector of the joint parameters,

- $\boldsymbol{Y}_{B o}$ and $\boldsymbol{Y}_{B j}$ are the coefficient matrices (or regressor matrices) of $\phi$,

- $\boldsymbol{Z}$ is the regressor matrix of $\boldsymbol{\xi}$ as follows:

$$
\boldsymbol{Z} \triangleq\left[\operatorname{diag}(\boldsymbol{u}) \quad \operatorname{diag}\left(\dot{\boldsymbol{q}}_{j}\right) \quad \operatorname{sgn}\left(\operatorname{diag}\left(\dot{\boldsymbol{q}}_{j}\right)\right)\right]
$$

Eq.(7) is the basic formulation used for identification. Though $\boldsymbol{\tau}_{c}$, strictly speaking, depends on the constraint forces acting on the joint axis, let us assume that the actuatorside friction is dominant and $\boldsymbol{\tau}_{c}$ is constant. When assuming that the geometric parameters like link lengths are known and $\phi_{B}$ and $\boldsymbol{\xi}$ are constant unknown values, Eq.(7) is linear with respect to the unknown parameters.

\section{B. Structual identifiability}

$\boldsymbol{\phi}_{B}$ and $\boldsymbol{\xi}$ can be identified uniquely, if Eq.(9) holds.

$$
\operatorname{dim} \mathrm{V}\left(\left[\begin{array}{ll}
\boldsymbol{Y}_{B o} & \boldsymbol{O} \\
\boldsymbol{Y}_{B j} & \boldsymbol{Z}
\end{array}\right]\right)=N_{B}+3 N_{J}
$$

where, notation $\operatorname{dim} \mathrm{V}(\boldsymbol{Y})$ means the dimension of the vector-space spanned by the column vectors of $\boldsymbol{Y}$. If in the case of a manipulator without external forces, $\phi_{B}$ cannot be identified uniquely from the equations of motion. The equations of a manipulator does not have the upper part of Eq.(4). As the actuator constants are regarded as unknown, then $\boldsymbol{Y}_{B j} \boldsymbol{\phi}_{B}+\boldsymbol{Z} \boldsymbol{\xi}=\mathbf{0}$ holds.

Let us premise that the humanoid system has $N_{J}$ DOF and $N_{J}+6$ generalized coordinates, and all the joints are fully actuated; all the elements of $\boldsymbol{q}, \dot{\boldsymbol{q}}, \ddot{\boldsymbol{q}}$ are linear independent. It is known that $\phi_{B}$ of a legged system can be identified only from the equations of the base-link; $\operatorname{dim} \mathrm{V}\left(\boldsymbol{Y}_{B o}\right)=N_{B}$ holds [12]. The necessary and sufficient condition of Eq.(9), therefore, is that Eq.(10) holds:

$$
\operatorname{dim} \mathrm{V}(\boldsymbol{Z})=3 N_{J}
$$

Eq.(10) means that the identifiability of whole system only depends on the modeling of actuators and joint frictions. In order to check the identifiability of the whole system with arbitrary actuators, we just check whether Eq.(10) holds. In the case of motors, it is obvious that Eq.(10) holds from Eq.(6), and therefore, Eq.(9) holds.

The identification model of Eq.(7), in other words, basically relies on the identification and identifiability of the equations of the base-link [12], and also allows us to identify the joint parameters simultaneously.

\section{Basic flow of identification}

Let us identify the parameters by using the following sensors:

- joint encoders which measure $\boldsymbol{q}_{j}$,

- a gyro and an accelerometer which obtain $\ddot{\boldsymbol{p}}_{o}$ and $\boldsymbol{\omega}_{o}$,

- force/moment sensors which detects $\boldsymbol{F}_{k}^{\text {ext }}$,

- motor current sensors measuring $\boldsymbol{u}$

where, $\ddot{\boldsymbol{p}}_{o}$ is the linear acceleration containing gravity acceleration with respect to the sensor coordinate, $\boldsymbol{\omega}_{o}$ is the angular velocity with respect to the sensor coordinate.

Let us select the link, which has the gyro and the accelerometer, as the base-link in Eq.(7). When representing the upper part Eq.(7) with respect to its local coordinate system, Eq.(7) does not include $\boldsymbol{q}_{0}, \dot{\boldsymbol{p}}_{0}$. Since $\ddot{\boldsymbol{p}}_{o}$ contains the gravity acceleration with respect to the local coordinate, $\boldsymbol{Y}_{B o}, \boldsymbol{Y}_{B j}, \boldsymbol{Z}, \boldsymbol{F}_{o}$ and $\boldsymbol{F}_{j}$ can be computed by $\boldsymbol{q}_{j}, \ddot{\boldsymbol{p}}_{o}, \boldsymbol{\omega}_{o}$, and their numerical derivatives. 
In order to identify the parameters, by sampling Eq.(7) from the sensor data, and we have:

$$
\left[\begin{array}{cc}
\widehat{\boldsymbol{Y}}_{B o}(t) & \boldsymbol{O} \\
\widehat{\boldsymbol{Y}}_{B c}(t) & \widehat{\boldsymbol{Z}}^{(t)}
\end{array}\right]\left[\begin{array}{c}
\boldsymbol{\phi}_{B} \\
\boldsymbol{\xi}
\end{array}\right]=\left[\begin{array}{c}
\widehat{\boldsymbol{F}}_{o}^{(t)} \\
\widehat{\boldsymbol{F}}_{j}(t)
\end{array}\right]+\left[\begin{array}{c}
\boldsymbol{e}_{o}^{(t)} \\
\boldsymbol{e}_{j}^{(t)}
\end{array}\right]
$$

where, notation $\widehat{\boldsymbol{x}}^{(t)}$ means the value which computed from $\boldsymbol{t}$-th sample of sensor data, and $\boldsymbol{e}_{o}$ and $\boldsymbol{e}_{j}$ are the errors.

The basic approach of the identification is the least squares method; we solve the problem such that:

$$
\min _{\boldsymbol{\phi}_{B}, \boldsymbol{\xi}} h=\sum_{t}\left(\boldsymbol{e}_{o}{ }^{(t) T} \boldsymbol{W}_{o} \boldsymbol{e}_{o}{ }^{(t)}+\boldsymbol{e}_{j}{ }^{(t) T} \boldsymbol{W}_{j} \boldsymbol{e}_{j}{ }^{(t)}\right)
$$

where, $\boldsymbol{W}_{o} \in \mathbb{R}^{6 \times 6}$ and $\boldsymbol{W}_{o} \in \mathbb{R}^{N_{J} \times N_{J}}$ are the weighting factors and the diagonal matrices. As the problem is the quadratic form, the analytical solution of the problem can be easily computed.

\section{Implementation}

When identifying the parameters, we often face the following problems:

- design of "persistent excitation (PE) trajectory" which can identify the whole parameters [18],

- inequality constraints about the physical consistency of the parameters [19].

Let us assume that PE trajectories cannot always be obtained, and let us know the a-priori knowledge which can be obtained, for example, from CAD data of the model. We fill the null-space of numerically unidentifiable parameters with the a-priori knowledge by the similar way as the method [9]. Instead of Eq.(12), we solve the following least squares with the a-priori knowledge:

$$
\begin{array}{r}
\min _{\boldsymbol{\phi}_{B}, \boldsymbol{\xi}} h+\left(\boldsymbol{\phi}_{B}-\boldsymbol{\phi}_{B}{ }^{r e f}\right)^{T} \boldsymbol{W}_{\phi}\left(\boldsymbol{\phi}_{B}-\boldsymbol{\phi}_{B}{ }^{\text {ref }}\right) \\
+\left(\boldsymbol{\xi}-\boldsymbol{\xi}^{\text {ref }}\right)^{T} \boldsymbol{W}_{\boldsymbol{\xi}}\left(\boldsymbol{\xi}-\boldsymbol{\xi}^{\text {ref }}\right)
\end{array}
$$

where, $\boldsymbol{W}_{\phi} \in \mathbb{R}^{N_{B} \times N_{B}}$ and $\boldsymbol{W}_{\boldsymbol{\xi}} \in \mathbb{R}^{3 N_{J} \times 3 N_{J}}$ are the weighting factors and the diagonal matrices. $\phi_{B}{ }^{r e f}$ and $\xi^{r e f}$ are the apriori knowledge. When it is difficult to obtain the a-priori knowledge like frictions, the values are set to zero.

Since the physical units of $\phi_{B}$ and $\boldsymbol{\xi}$ are different, the weighting factors has to be normalized among them. Here is the example to design the weighting factors:

$$
\begin{aligned}
& \boldsymbol{W}_{\phi}=w_{\phi} \sigma_{\max }\left(\Lambda_{\phi}\right) \boldsymbol{I} \\
& \boldsymbol{W}_{\xi}=\operatorname{diag}\left(\left[\begin{array}{c}
w_{T} \sigma_{\max }\left(\Lambda_{T}\right) \mathbf{1}_{N_{j}} \\
w_{d} \sigma_{\max }\left(\Lambda_{d}\right) \mathbf{1}_{N_{j}} \\
w_{s} \sigma_{\max }\left(\Lambda_{s}\right) \mathbf{1}_{N_{j}}
\end{array}\right]\right) \\
& \boldsymbol{\Lambda}_{\phi} \triangleq \sum_{t}\left(\left[\begin{array}{ll}
\widehat{\boldsymbol{Y}}_{B o}{ }^{(t) T} & \widehat{\boldsymbol{Y}}_{B j}{ }^{(t) T}
\end{array}\right] \boldsymbol{W}_{o}\left[\begin{array}{l}
\widehat{\boldsymbol{Y}}_{B o}{ }^{(t)} \\
\widehat{\boldsymbol{Y}}_{B j}{ }^{(t)}
\end{array}\right]\right) \\
& \boldsymbol{\Lambda}_{T} \triangleq \sum_{t}\left(\operatorname{diag}\left(\boldsymbol{u}_{t}\right)^{2}\right) \\
& \boldsymbol{\Lambda}_{d} \triangleq \sum_{t}\left(\operatorname{diag}\left(\dot{\boldsymbol{q}}_{t}\right)^{2}\right) \\
& \boldsymbol{\Lambda}_{c} \triangleq \sum_{t}\left(\operatorname{sign}\left(\operatorname{diag}\left(\dot{\boldsymbol{q}}_{t}\right)\right)^{2}\right)
\end{aligned}
$$
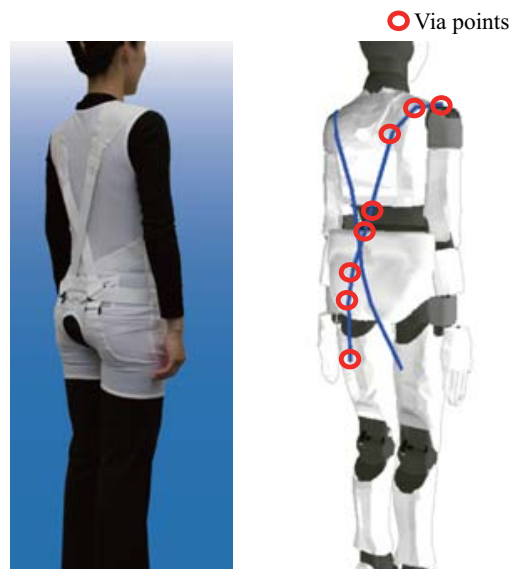

Fig. 2. Overview of assistive wear "Smart Suite Lite" (Left) and its computational wire model (Right). There are two wires which respectively have eight via points attached on the robot.

where, $\mathbf{1}_{n} \in \mathbb{R}^{n \times 1}$ is the vector whose elements are all one, $\sigma_{\max }(\boldsymbol{\Lambda})$ returns the maximum singular value of $\boldsymbol{\Lambda}$, and the normalized weighting factors are $\boldsymbol{w}_{\phi}, \boldsymbol{w}_{T}, \boldsymbol{w}_{d}$ and $\boldsymbol{w}_{c}$.

\section{IDENTIFICATION OF PASSIVE POWER-ASSIST DEVICES}

\section{A. General formulation of wire-based assistive devices}

Nominal modeling of supporting mechanism of assistive devices has a great role in order to design, modify, and evaluate them. If a passive power-assist device consists of several elastic bands or belts, the supporting torques generated in human joints can be modeled by the same manner as the formulation of wire-driven multi-body systems. It is also related to the musculoskeletal analysis; the joint torques are generated by several muscles modeled as elastic wires [3]. We, therefore, can utilize the same formulation and framework which map the elastic forces to the joint torques, and prepare elastic model of each assistive device.

Each wire has several via points fixed on the rigid-body system. The supporting torques are formulated as follows:

$$
\boldsymbol{\tau}_{s}=\sum_{i} \boldsymbol{J}_{i}^{T} f_{i}
$$

where,

- $\boldsymbol{N}_{l}$ is the number of wires,

- $\boldsymbol{N}_{v, i}(>1)$ is the number of via points of $i$-th wire,

- $\boldsymbol{\tau}_{s} \in \mathbb{R}^{N_{J}}$ is the vector of supporting torques,

- $f_{i} \in \mathbb{R}$ is the elastic force of $i$-th wire,

- $\boldsymbol{J}_{i} \in \mathbb{R}^{1 \times N_{J}}$ is the Jacobian matrix of length $l_{i}$ of $i$-th wire as follows:

$$
\begin{aligned}
& \boldsymbol{J}_{i} \triangleq \frac{\partial l_{i}}{\partial \dot{\boldsymbol{q}}_{J}} \\
& l_{i} \triangleq \sum_{j=2}^{N_{v, i}}\left\|\boldsymbol{p}_{i, j}-\boldsymbol{p}_{i, j-1}\right\|
\end{aligned}
$$

- $\boldsymbol{p}_{i, j} \in \mathbb{R}^{3}$ is the position of $j$-th via point of $i$-th wire. 

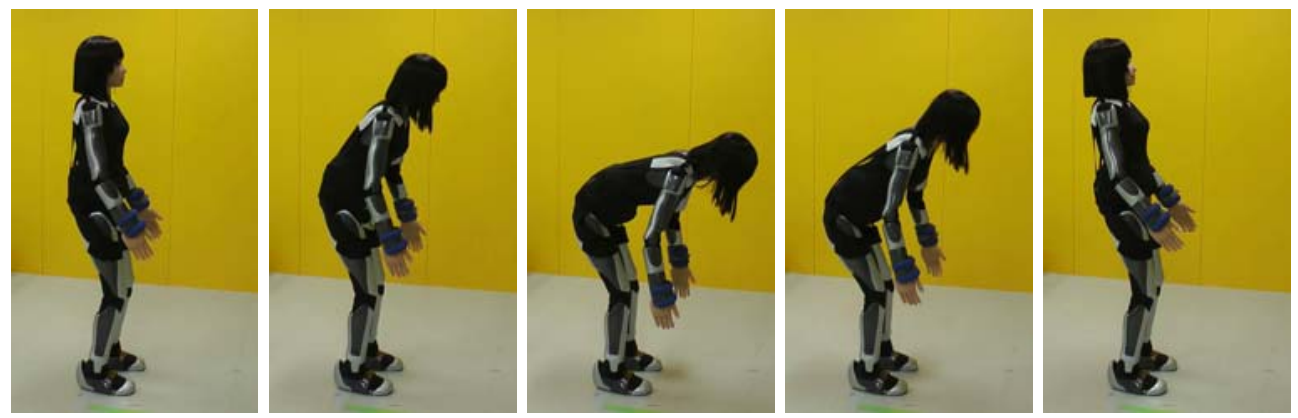

Fig. 3. Snapshots of lifting motion of HRP-4C

\section{B. Modeling and identification of the elastic model of SSL}

The basic function of "Smart Suit Lite" shown in Fig.2 (left) is to reduce the torque at the lower back by stretched two elastic bands fixed at the shoulders and thighs. In order to represent the two SSL bands, eight via points per one band are located on the surface of the model of HRP-4C as shown in Fig.2 (right). Each via point is fixed on the corresponding link, and its position can be computed by the forward kinematics computation.

The elastic force of each SSL band is formulated as a linear spring as follows:

$$
f_{i}= \begin{cases}-k_{i}\left(l_{i}-l_{0, i}\right) & \left(l \geq l_{0, i}\right) \\ 0 & (l<0)\end{cases}
$$

where, $l_{i 0}$ is the natural length and $k_{i}$ is the stiffness of SSL band $i$. The stiffness of each SSL band is $k_{i}=197.8[\mathrm{~N} / \mathrm{m}]$, which is identified by the experiment.

The supporting torques of SSL are computed from Eq.(20) and Eq.(23). If the geometric parameters of the robot and the location of via points are known, $\boldsymbol{\tau}_{s}$ depends on the following constant parameters:

- stiffness $k_{i}$

- natural length $l_{0, i}$

Let us identify $k_{i}$ and $l_{0, i}$ from the estimated supporting torques. The identification problem of them is written as:

$$
\min _{\forall i k_{i}, l_{0, i}} \sum_{t}\left(\left\|\widehat{\boldsymbol{\tau}}_{s}^{(t)}-\widehat{\boldsymbol{J}}_{i}^{(t)} \widehat{f}_{i}^{(t)}\right\|\right)
$$

where, $\widehat{\tau}_{s}(t)$ is the vector of the supporting torque at sample $t$, which is estimated from Eq.(2) or Eq.(3). $\widehat{\boldsymbol{J}}_{i}^{(t)}$ is computed by $\boldsymbol{q}_{j}{ }^{(t)}$ and independent from $k_{i}$ and $l_{0, i} \cdot \widehat{f}_{i}(t)$ is made of not only $\boldsymbol{q}_{j}{ }^{(t)}$ but also $k_{i}$ and $l_{0, i}$.

As can been seen from Eq.(23), $f_{i}$ is not linear with respect to $l_{i, 0}$ because of the case statements. Eq. (24) is not the quadratic problem unlike Eq.(12) and Eq.(13). Since Eq.(24) has no equality or inequality constraints, the problem can be solved by, for example, the quasi-Newton method.

\section{EXPERIMENTS}

\section{A. Human motion measurement and retargeting}

The basic pilot experiments were conducted with HRP4C wearing SSL. The evaluation flow described in Section II was validated.
In step 1(S1), we prepared a motion of bending forward from the waist holding a dumbbell $(0.5 \mathrm{~kg}$ each $)$ in the both hands. It is because SSL supports stretching the muscles of the lower back, and is expected to be effective when wearer is bending down for a long time or repeatedly. The motion was acquired by Vicon Motion Systems. Sampling rate was 200 frames per second. More detailed information about the measurement setup of motion capture is shown in [4]. By utilizing the motion retargeting method [7], in step 2, the measured lifting motion was converted to a trajectory feasible to HRP-4C. The snapshots of the converted motion are shown in Fig.3.

\section{B. Identification of $H R P-4 C$}

We identified the inertial parameters and joint parameters of HRP-4C by solving Eq.(13) in step 3. the converted trajectories were performed by HRP-4C without SSL. One of them was chosen in order to identify the parameters. The a-priori parameters used in Eq.(12) were the inertial parameters provided by the manufacturer; we don't model the dumbbells in the both hands in advance. Though the electronic current and the torque constant of each motor could not be accessed, the information of the joint torque was provided. We regarded the provided values as $\boldsymbol{u}$, and the apriori values of $\boldsymbol{k}_{T}$ is equal to $\mathbf{1}_{N_{J}}$. Here are the configuration of weighting factors: $\boldsymbol{w}_{\phi}=0.1 / \sqrt{N_{B}}, \boldsymbol{w}_{T}=0.1 / \sqrt{N_{J}}, \boldsymbol{w}_{d}=$ $0.01 / \sqrt{N_{J}}$ and $\boldsymbol{w}_{c}=0.01 / \sqrt{N}_{J}$.

Fig.4 and Fig.5 show the errors between the measured forces and those estimated from the model: $\boldsymbol{e}_{0}$ or $\boldsymbol{e}_{j}$ defined in Eq.(11). Fig.4 shows the results of the pitch-axis moment of the base link (the waist link), and Fig.5 shows the torque error of the pitch joint of the torso, which is expected to be mostly supported by SSL. The red lines show the error when using the identified parameters, and blue lines show the error when using the a-priori parameters: the values provided by the manufacturer. The black dotted lines are the moment and the joint torque measured from the sensors, which are plotted in order to check the scale of the data. As can be seen from the figures, the identified parameters show better performance rather than the a-prior parameters.

\section{Estimation of the supporting torques of SSL}

The converted trajectories were performed by HRP-4C with and without SSL respectively. The supporting torques 


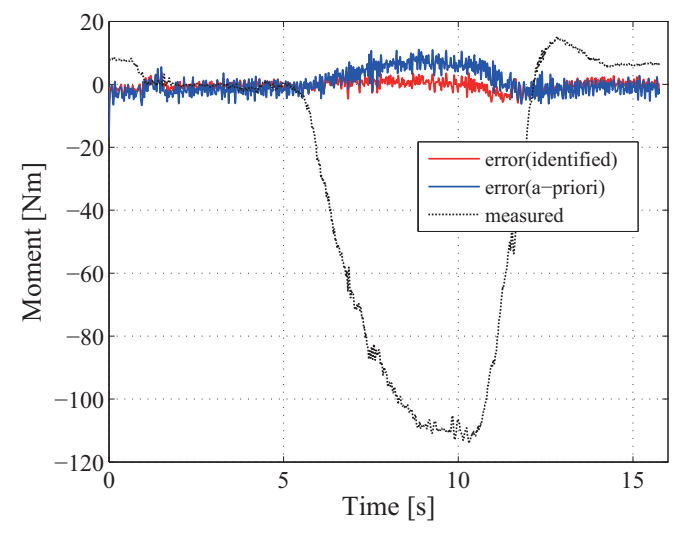

Fig. 4. Direct validation of the errors about the moment along pitch-axis of the base link (waist link): the error when using the identified parameters (red), the error with the a-priori parameters (blue), and the measured external moment (black). The root mean square error (RMSE) of the red line is 1.58 $\mathrm{Nm}$, and that of the blue line is $3.82 \mathrm{Nm}$.

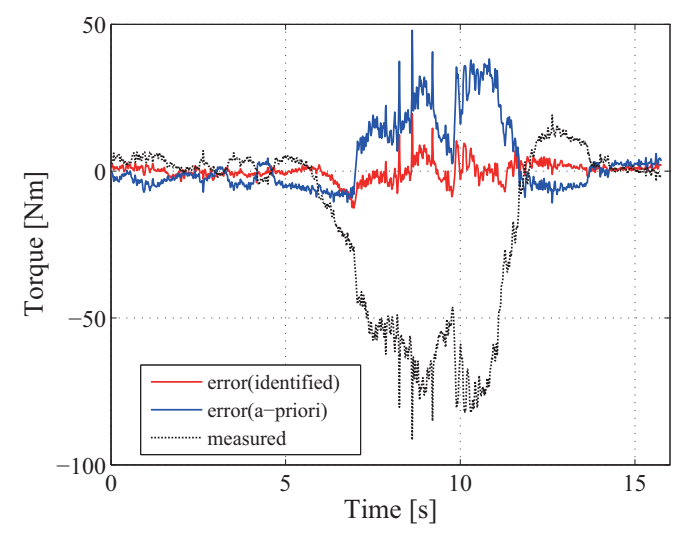

Fig. 5. Direct validation of the errors about the torque of the pitch joint of the torso: the error when using the identified parameters (red), the error with the a-priori parameters (blue), and the measured joint torque (black). The RMSE of the red line is $3.06 \mathrm{Nm}$, and that of the blue line is 12.50 $\mathrm{Nm}$.

of SSL were estimated by the two approaches of step 4, reproduced as follows:

(A). Compute the difference of joint torque between with and without the device: Eq.(2)

(B). Compute the difference between the measured torque and the torque estimated from the model: Eq.(3)

Note that approach (B) has an advantage that it can be applied in cases where the motions with and without supportive devices are not quite similar, since we only use the motions with the device.

Fig.6 shows the comparison of the supporting torques of the pitch joint of the torso. The red line shows the supporting torque estimated by approach (A) with the identified values, and the blue line is the torque estimated by approach (B) with the identified values. The green line represents the torque estimated by approach (B) with the a-priori values. The black line shows the torque estimated from the SSL simulation

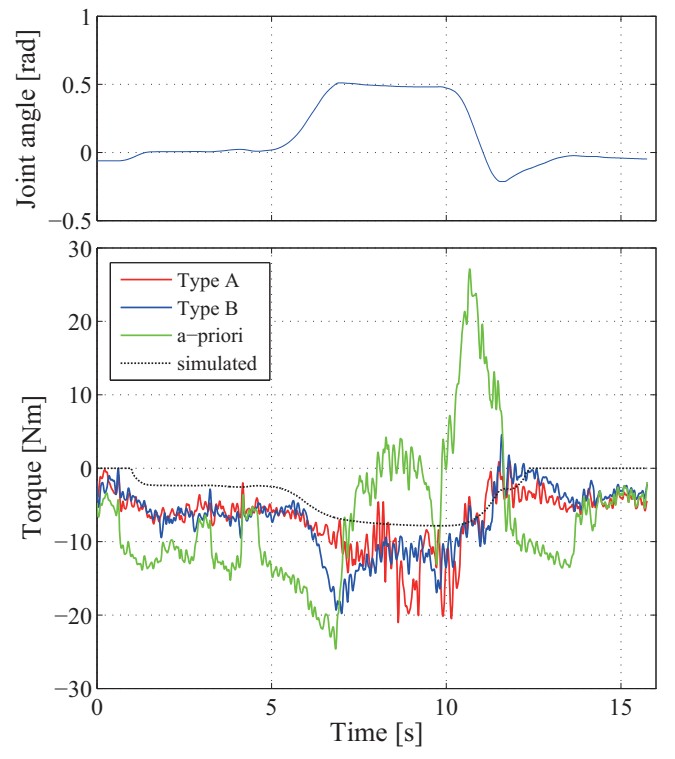

Fig. 6. Comparison of the torso joint torques: by approach (A) using the identified values (red), by approach (B) using the identified value (blue) and using the a-priori parameters (green), and by the SSL simulation with the ground truth stiffness (black).

described in Section IV. As the initial length of each SSL band is unknown, we regarded the length at the initial posture as the initial one.

Though there exists an offset, the red and blue lines shows the good correlation with the black line; on the other hand, the green line does not match the black line at all. The results shows the supportive effect at the torso joint during the bending motion. As the black lines does not mean the measured values because of the lack of the natural length, the comparison between approach (A) and (B) will be shown in step 5 .

\section{Identification of SSL}

We evaluated the mechanical properties of SSL by using the supporting torques estimated from the sensors with the identification (Fig.6). The stiffness and the natural length of each SSL band were identified by solving Eq.(24). We added the constraint such that the stiffness of each wire is same because of the symmetry; on the other hand, the natural lengths are different among the wires. Table I shows the result of the identified stiffness of the SSL band; (a) the measured value, (b) the identified value by using the supporting torque estimated from approach (A), (c) the identified one by approach (B), and (d) the identified one by using the torque estimated from the a-priori values.

Table I shows that the proposed scheme could successfully identify the mechanical property of the device. The results also indicate that the identification has a great role when we evaluate the device with a humanoid robot. The error of the identified value in case (A) is $5 \%$, and the error in case (B) is about $20 \%$. Though there is the strict constraint such that the same motion has to be performed between with and without the device, the case (A) showed better performance 
TABLE I

COMPARISON OF THE STIFFNESS OF THE SSL BAND.

\begin{tabular}{|l||c|}
\hline & stiffness[N/m] \\
\hline (a) measured value & 197.8 \\
(b) identified by approach (A) & 187.0 \\
(c) identified by approach (B) & 242.2 \\
(d) identified with a-priori model & 55.9 \\
\hline
\end{tabular}

than case (B). The error of case (B) is not small currently. That is mainly because the friction model cannot eliminate the effect of the sudden change between kinetic to static frictions; there is the error peak when the joint stops in Fig.6. To exploit the advantage of approach (B) which needs only the motions with devices, improvements of modeling such as friction models will be addressed in our future work. In this paper, only a small data-set for identification was needed; the parameters need not be fully excited, because the same motion could be performed between with and without the device. The problem to generate PE trajectories will also be investigated in our future work.

\section{CONCLUSION}

This paper presented the application of a humanoid robot as an evaluator of assistive devices, and proposed a framework of the evaluation by utilizing identification of mechanical properties of a humanoid robot. We also showed the following techniques useful for our application:

- A method for identifying simultaneously the parameters of the whole body dynamics, the actuator model, and the friction model.

- A general modeling to compute the supporting torques of passive assistive wears by using the formulation of wire driven multi-body systems.

We evaluated the supporting torques of a passive assistive wear "Smart Suit Lite (SSL)" as an example of assistive device, and utilized HRP-4C as the humanoid platform.

After identifying the mechanical properties of HRP-4C, the supporting torques were estimated by the two approaches: (A) by computing the difference of joint torque between with and without the device, and (B) by computing the difference between the measured torque and the torque estimated from the identified model. In both cases, the estimated supporting torques show the good correlation with the simulation result of SSL with the ground truth stiffness.

The estimated supporting torques were also used to identify the stiffness of the device as an example of the evaluation. The identified stiffness is validated and compared to the ground truth value. The error of the identified value in case (A) was $5 \%$, and the error in case (B) was about $20 \%$; on the other hand, the error without identification is about $70 \%$. The proposed scheme could successfully identify the mechanical property of the device, which implies that the identification has an important role when we evaluate the device with a humanoid robot. The case (A) showed better performance than case (B), because there is the strict constraint in case (A) such that the same motion has to be performed between with and without the device. The error of case (B) is not small currently. To exploit the advantage of approach (B) which needs only the motions with devices, improvements of modeling such as friction models will be addressed in our future work.

\section{REFERENCES}

[1] Y. Sankai, "Hal: Hybrid assistive limb based on cybernics," in Robotics Research, Springer Tracts in Advanced Robotics, vol. 66. Springer, 2011.

[2] Y. Imamura, T. Tanaka, Y. Suzuki, K. Takizawa, and M. Yamanaka, "Motion-based-design of elastic material for passive assistive device using musculoskeletal model," J. of Robotics and Mechatronics, vol. 23, no. 6, pp. 58-66, 2011

[3] Y. Nakamura, K. Yamane, and I. Suzuki, "Somatosensory computation for man-machine interface from motion-capture data and musculoskeletal human model," IEEE Transactions on Robotics, vol. 21, no. 1, pp. 58-66, 2005

[4] K. Miura, E. Yoshida, Y. Kobayashi, Y. Endo, F. Kanehioro, K. Homma, I. Kajitani, Y. Matsumoto, and T. Tanaka, "Humanoid robot as an evaluator of assistive devices," in Proc. of the IEEE Int. Conf. on Robotics and Automation, 2013, pp. 671-677.

[5] K. Kaneko, F. Kanehiro, M. Morisawa, K. Miura, S. Nakaoka, and S. Kajita, "Cybernetic human hrp-4c," in Proc. of the IEEE-RAS Int. Conf. on Humanoid Robots, Dec 2009, pp. 7-14.

[6] S. Nakaoka, A. Nakazawa, F. Kanehiro, K. Kaneko, M. Morisawa, H. Hirukawa, and K. Ikeuchi, "Learning from observation paradigm: Leg task models for enabling a biped humanoid robot to imitate human dances," Int. J. of Robotic Research, vol. 26, no. 8, pp. 829-844, 2007.

[7] K. Miura, M. Morisawa, S. Nakaoka, F. Kanehiro, K. Harada, K. Kaneko, and S. Kajita, "Robot motion remix based on motion capture data towards human-like locomotion of humanoid robots," in Proc. of the IEEE-RAS Int. Conf. on Humanoid Robots, Dec 2009, pp. 596-603.

[8] K. Yamane and Y. Nakamura, "Dynamics computation of structurevarying kinematic chains and its application to human figures," IEEE Trans. on Robotics and Automation, vol. 16, no. 2, pp. 124-134, 2000

[9] G. Venture, K. Ayusawa, and Y. Nakamura, "Optimal estimation of human body segments dynamics using realtime visual feedback," in Proc. IEEE/Int. Conf. on Intelligent Robot System, 2009, pp. 1627 1632.

[10] P-L. Santaguida, M. Pierrynowski, C. Goldsmith, and G. Fernie, "Comparison of cumulative low back loads of caregivers when transferring patients using overhead and floor mechanical lifting devices," Clinical Biomechanics, vol. 20, no. 9, pp. 906-916, 2005.

[11] A. Albu-Schäffer, S. Haddadin, Ch Ott, A. Stemmer, T. Wimböck, and G. Hirzinger, "The DLR lightweight robot: design and control concepts for robots in human environments," Industrial Robot, vol. 34, no. 5, pp. 376-385, 2007.

[12] K. Ayusawa, G. Venture, and Y. Nakamura, "Identifiability and identification of inertial parameters using the underactuated base-link dynamics for legged multibody systems," Int. J. of Robotics Research, vol. 33, no. 3, pp. 446-468, 2014.

[13] K. Yoshida, D.N. Nenchev, and M. Uchiyama, "Moving base robotics and reaction management control," in Proc. of the 7th Int. Symp. of Robotics Research, 1995, pp. 100-109.

[14] H. Mayeda, K. Osuka, and A. Kanagawa, "A new identification method for serial manipulator arms," in Proc. of 9th IFAC World Congress, 1984, vol. 2, pp. 74-79.

[15] C.-G. Atkeson, C.-H. An, and J.-M. Hollerbach, "Estimation of inertial parameters of manipulator loads and links," Int. J. of Robotic Research, vol. 5, no. 3, pp. 101-119, 1986.

[16] H. Mayeda, K. Yoshida, and K. Osuka, "Base parameters of manipulator dynamic models," IEEE Trans. on Robotics and Automation, vol. 6, no. 3, pp. 312-321, 1990.

[17] W. Khalil and F. Bennis, "Symbolic calculation of the base inertial parameters of closed-loop robots," Int. J. of Robotics Research, vol. 14, no. 2, pp. 112-128, 1995.

[18] M. Gautier and W. Khalil, "Exciting trajectories for the identification of base inertial parameters of robots," Int. J. of Robotics Research, vol. 11, no. 4, pp. 363-375, 1992.

[19] K. Yoshida and W. Khalil, "Verification of the positive definiteness of the inertial matrix of manipulators using base inertial parameters," Int. J. of Robotics Research, vol. 19, no. 5, pp. 498-510, 2000. 